\title{
APROXIMACIÓN A LA FISONOMÍA DEL TERRENO CULTIVADO Y A LAS PRÁCTICAS AGRÍCOLAS EN LA RIBEIRA SACRA DURANTE LOS SIGLOS XIV Y XV. ${ }^{1}$
}

\section{APPROXIMATION TO THE PHYSIOGNOMY OF THE CULTIVATED AREA AND TO THE AGRICULTURAL PRACTICES IN THE RIBEIRA SACRA DURING THE XIVTH AND XV ${ }^{\mathrm{TH}}$ CENTURY.}

\author{
JOSÉ ANTONIO LÓPEZ SABATEL \\ Unversidad de Santiago de Compostela
}

\begin{abstract}
Resumen
Este artículo pretende un acercamiento a lo que debió ser el semblante físico y extensión de los terrenos ganados en beneficio de la actividad agrícola en el seno del área de influencia de cinco cenobios situados a ambos márgenes del río Sil en el tramo precedente a su desembocadura en el Miño.

También se centrará en las diversas prácticas agrícolas destinadas al cuidado de la castaña y la vid así como al trabajo en las tierras de cereal. Prácticas éstas que en lo relativo al sistema de cultivos tendrán incidencia en la fisonomía del espacio agrario.
\end{abstract}

\section{Palabras clave:}

Ribeira Sacra, Sociedad feudal, Baja Edad Media, Historia Rural,

\begin{abstract}
This article tries an approach to which it had to be the physical semblante and extension of terrain taken in benefit of the agricultural activity in the area of influence of five monasteries located to both margins of the Sil river in the preceding section to his opening in the Miño. Also it will be centered in the diverse agricultural practices destined to the care of the chestnut and the grapevine as well as to the work in territories of cereal. Practices these that with respect to the system of cultures will have incidence in the appearance of the agrarian space.
\end{abstract}

\section{Keywords}

Sacred Ribeira, feudal Society, Low Average Age, Rural History, agrarian Landscape, agricultural Practices.

\footnotetext{
${ }^{1}$ Entregado el 20 de febrero de 2007.
} 


\section{FISONOMÍA DEL TERRENO CULTIVADO}

\section{Los viñedos}

Es sabido que una limitación de la documentación eclesiástica es la que se infiere de la total ausencia de datos estadísticos ${ }^{2}$ tanto referidos a la metrología de la superficie como a cualquier otro tipo de precisión numérica ${ }^{3}$. Circunstancia que originará, entre otras cosas, una mengua correspondiente a menciones directas referidas a la extensión de las tierras cultas y en especial a las dedicadas al cereal sujetas a medidas no reales de superficie sino de sembradura.

Mayor fortuna se puede hallar en relación con las explotaciones relativas al viñedo. Éstas, se medirán por cavaduras o lo que es lo mismo la porción de tierra que un hombre puede trabajar en una jornada ${ }^{4}$. Espacio por consiguiente que no tiene nada de absoluto ya que depende del esfuerzo humano.

Sin embargo, en pocas ocasiones se explicitan en la documentación las cavaduras que pueden llegar a comprender una viña y en la mayoría de los casos cuando esto sucede los terrenos mencionados se circunscribirán al actual municipio de Pantón donde hoy día el viñedo se mesura por ferrados y jornales de viñedo según las parroquias.

De manera que en la feligresía de Pombeiro localizaremos

...Conven a saber que vos aforamos huna nosa leyra de viña a que chaman do Casar... et outrosy vos aforalos outra leyra a que chaman do Poombar....as quaes ditas duas leyras jazen anbas en a Cabreyra sub o signo do dito noso moesteiro, et seeren huna per outra fasta dez omees cavadura... ${ }^{5}$

...Item vos aforamos duas cabaduras de viña con seus castanos huna en no val d'Amande, que parte con a viña d'Afonso Carneyro, con outra que foy de Gonçalvo Ferreiro; et outra cabadura que jaz en Avoos, que parte con huna d'Afonso Carneyro et de Diego Sileyro... ${ }^{6}$

Teniendo en cuenta que tradicionalmente, como con anterioridad se señaló, la cavadura supondrá la jornada de trabajo de un hombre ${ }^{7}$ y que en la feligresía de Pom-

\footnotetext{
${ }^{2}$ PORTELA SILVA, Ermelindo: "Propiedad y formas de explotación de la tierra en la Galicia medieval" en La economía agraria en la historia de España. Propiedad, explotación, comercialización, rentas, Madrid, Alfaguara (1979), p. 124.

${ }^{3}$ PALLARES MÉNDEZ, M ${ }^{\mathrm{a}}$ del Carmen: El monasterio de Sobrado: un ejemplo de protagonismo monástico en la Galicia medieval, A Coruña, Diputación Provincial de A Coruña, 1975, pp. 44.

${ }^{4}$ SÁNCHEZ CARRERA, Ma del Carmen: El Bajo Miño en el siglo XV. El espacio y los hombres, A Coruña, Fundación Pedro Barrie de la Maza, 1997, p.117.

${ }^{5}$ Doc 205., CDP., p. 278.

${ }^{6}$ Doc 244., CDP., p. 312.

${ }^{7}$ CDP. Doc 96: ... Outrosy vos aforamos o monte de Soutonovo en quanto ponades quatro omees cavadura de viña onde for mellor para ela...., pp. 154-155.
} 
beiro no se usa el jornal sino el ferrado de viñedo que equivale a 5,09 áreas ${ }^{8}$ se puede colegir, dada la similitud de superficie respecto a la cavadura de otras zonas al margen izquierdo del Sil, junto con la convicción de que una jornada de trabajo no ha de variar en demasía entre ambas riberas, la correspondencia entre cavadura y ferrado en lo que respecta a los montes de esta parroquia en el actual municipio de Pantón.

Siguiendo con esta equivalencia cabadura-ferrado de viñedo se deduce que las dos leiras de viña situadas en Cabreira tendrán una extensión entre las dos de 5.090 $\mathrm{m}^{2}$ casi media hectárea mientras que tanto la viña ubicada en Amande como la hallada en Avoos (un antiguo casal hoy sin localizar) medirán $509 \mathrm{~m}^{2}$ respectivamente.

La parroquia de Santiago de Cangas compartirá la metrología de Pombeiro en relación con el viñedo, de esta manera cuando el abad del monasterio de San Esteban de Ribas de Sil afora la viña llamada O Prado sita en dicha feligresía y conmina al forero a que

...et he posto que desta dita vina que non labredes mays de tres omees cavadura....9

la superficie que se destinará a la producción vitivinícola será de $1527 \mathrm{~m}^{2}$.

En la parroquia de Santiago de Castillón el ferrado de viñedo coincidirá plenamente con el valor más frecuente otorgado a la cavadura tanto en la provincia de Orense como en la de Pontevedra: 4,37 áreas ${ }^{10}$. En consecuencia, la equiparación ferrado-cavadura en esta zona será aún más nítida si cabe que en las parroquias de Pombeiro y Cangas.

...conbén a saber que vos aforamos como dito he a viña de Cas da Alonso... que son seys cabaduras... ${ }^{11}$

Así pues, serán $2622 \mathrm{~m}^{2}$ los que marquen la extensión de esta viña enmarcada en Santiago de Castillón.

Más dudas surgirán al adentrarnos en las parroquias de San Martiño de Pantón, Santa María de Ferreira y San Mamede de Mañente ya que en ellas, hoy día, se utilizará no el ferrado sino el jornal de viña cuyo valor será el de medio ferrado que

LÓPEZ CARREIRA, Anselmo: "O traballo da viña nun documento ourensán do século XIV”en Boletín Auriense, 18-19 (1988-89), pp. 155-167/ 162.

${ }^{8}$ FERNÁNDEZ JUSTO, M ${ }^{\mathrm{a}}$ Isabel: La metrología tradicional gallega. Aportación a los estudios sobre el medio rural, vol 1, Madrid, Instituto Geográfico Nacional. Centro español de metrología, 1986, p. 244.

${ }^{9}$ Doc 183., CDE., p. 389.

${ }^{10}$ FERNÁNDEZ JUSTO, $\mathrm{M}^{\mathrm{a}}$ Isabel: La metrología tradicional gallega. Aportación a los estudios sobre el medio rural, vol 1, Madrid, Instituto Geográfico Nacional. Centro español de metrología, 1986, pp. 67 y 73.

${ }^{11}$ Doc 218., CDF., p. 279. 
en estas localidades corresponderá a 2,18 áreas ${ }^{12}$. Es por ello que sea más que factible que una cavadura de la época estudiada equivalga a dos jornales de viña o lo que es lo mismo 4,36 áreas aproximadamente la estimación estándar de cavadura en las provincias de Orense y Pontevedra.

...e a nosa viña que jaz en Cas Franco e son d'oyto omees cavadura, e con a viña dos Moradouros que son çinquo omees cavadura e con outras viñas que jazen a Avelaayra que son tres omees cavadura... ${ }^{13}$

La documentación aquí mencionará dos viñas situadas en la parroquia de Pantón con una superficie de 3488 y $2180 \mathrm{~m}^{2}$ para cada una de ellas, a la vez que hace referencia a otras viñas sin especificar su número y sitas en Ferreira que ocuparán $1308 \mathrm{~m}^{2}$ de terreno.

Y en Mañente ya en el siglo XVI

...conbén a saber que vos aforo o terreo da Fonte, que serán dúas cabaduras de viña, que demarca de todos los cavos con el dicho Juan de Agronogueyras; e más otro tereo que jaz ao castelo vello, que será una cabadura de vyna... ${ }^{14}$

nos hallamos ante dos terrenos de viña, uno de $872 \mathrm{~m}^{2}$ y otro de $436 \mathrm{~m}^{2}$.

$\mathrm{Al}$ abandonar el actual municipio de Pantón para adentrarnos en tierras de $\mathrm{O}$ Saviñao la documentación reflejará dos menciones relativas a la extensión del viñedo en la parroquia de Santa Mariña de Rosende

.... a viña da Rancada, según que a vos tragedes a jur et a mao... et que britades as matelas que en ela estan, que son tres cavaduras... ${ }^{15}$

Mientras, Alfonso Pernas aforará al clérigo de Santa Marta de Velle diez cavaduras de viña en la cortiña de la granja de Rosende. ${ }^{16}$

La dificultad viene dada en cuanto que el jornal de viñedo en esta feligresía comprende $3,14 \mathrm{~m}^{2}$. Por lo que siendo coherentes con los casos anteriores y ejecutando la correspondencia 1 cavadura $=1$ ferrado de viñedo $=2$ jornales de viñedo, la cavadura en esta zona alcanzaría los $628 \mathrm{~m}^{2}$, superficie distante tanto de los $509 \mathrm{~m}^{2}$ de Pombeiro y Cangas, los $437 \mathrm{~m}^{2}$ de Castillón y los $436 \mathrm{~m}^{2}$ de Ferreira, Pantón y Mañente.

12 FERNÁNDEZ JUSTO: La metrología tradicional, vol. I, pp. 57 y 248.

${ }^{13}$ Doc 125., CDP., p. 197.

${ }^{14}$ Doc 220., CDF., p. 282.

${ }^{15}$ Doc 229., CDP., p. 298.

${ }^{16}$ Doc 223., CDE., p. 403. 
Teniendo en cuenta que el jornal de viñedo en Rosende coincide con la estipulación de cavadura que realiza Bouhier en su magno estudio geográfico sobre Galicia $^{17}$ que además es utilizado por López Carreira en su artículo acerca del trabajo de la viña en Orense ${ }^{18}$ y ante el riesgo de pecar por defecto antes que por exceso, usaré al igual que estos dos autores, el valor de la cavadura en correspondencia con el jornal de viñedo, o lo que es lo mismo, las citadas 3,14 áreas.

De esta manera, una de las viñas de Rosende no superará los 1000 m² $\left(942\right.$ m²$\left.^{2}\right)$ mientras que otra rebasará los $3000 \mathrm{~m}^{2}\left(3.140 \mathrm{~m}^{2}\right)$.

Dejando la provincia de Lugo se concretará el aforamiento de cinco y diez cavaduras de viña en Velle en las proximidades de Orense ${ }^{19}$ es decir 2185 m $^{2}$ y $4370 \mathrm{~m}^{2}$ respectivamente.

En cuanto a su distribución y morfología la viña constituirá un elemento sin el cual sería imposible imaginar el paisaje agrario de esta zona. Un paisaje bastante fragmentado si se tiene en consideración el gran número de lindes que separan unas viñas de otras. De forma, que como sucederá en la vecina región del Bierzo estudiada por M. ${ }^{a}$ del Carmen Rodríguez González ${ }^{20}$, de estas parcelaciones se deduce una distribución homogénea del viñedo a lo largo de los espacios donde este cultivo está consolidado como el de mayor relevancia con un especial protagonismo en la Tierra de Lemos. ${ }^{21}$

Así, será necesario para su explotación que las viñas queden perfectamente marcadas et dibisadas $^{22}$. En previsión de los posibles estragos que pudiera cometer el ganado o incluso la mano del hombre en periodo de vendimia el viñedo hay que

${ }^{17}$ BOHUIER, Abel: Ensaio xeográfico de análise e interpretación dun vello complexo agrario, vol II, s.1., Xunta de Galicia, 2001, pp. 1278-1270.

${ }^{18}$ LÓPEZ CARREIRA, Anselmo: “O traballo da viña nun documento ourensán do século XIV”en Boletín Auriense, 18-19 (1988-89), pp. 155-167/ 162.

19 Docs 200 y 218., CDPR., pp. 243 y 248.

${ }^{20}$ RODRÍGUEZ GONZÁLEZ, M. ${ }^{\mathrm{a}}$ Carmen: Economía y poder en el Bierzo del siglo XV. San Andrés de Espinareda, Santiago de Compostela, Universidad de Santiago, 1992, p. 118.

${ }^{21}$ CDF. Doc 57: ... hua leira de viña que nos avemos no Outeiro de teiro de Gistara...que jaz cabo outra leyra do dito Johán Pérez, a qual jaz cabo hua que foy de Domingo Vidal e de Fernán Díaz de Villagilde. Et vos o dito Johán Perez dardes a nos e ao dito moesteiro outra leira de viña... cabo outra nosa leira e do dito moesteiro, a qual jaz cabo hua que tina Afonso Eanes...., p. 73.

CDF. Doc 67: ...conbén a saber, dous leyros de vynas que jazen en Balado, entre hua do mosteiro e outra de Johan Fernández de Goyán...., p.86.

CDF. Doc 101: ...vendemos a vos... hun meu leiro de vyna que jas en Outar de Gistara, ontre hun de Fernán Lourenço e outro que lavra Afonso Rey, asy commo vay da vyna que lavra Juan Crismente e vay topar en outra vyna de Fernan Ribadal...., p. 101.

CDP. Doc 103: ...conven a saber, que vos vendo o meu leiro de viña que eu tenno en foro do moesteiro de Poombeiro, o qual leiro jaz entre huna viña de Vasco Fernández da Millara e outra de Vasco de Robadal, e en fondo topa con un leiro de Alonso Rey...., p. 175.

22 Doc 126., CDE., p. 358. 
"taparlo" 23 y cerrarlo bien ${ }^{24}$, bien por medio de un vallado ${ }^{25}$, de paredes $^{26}$ y de muros. $^{27}$

Las extensiones vitivinícolas se expandirán por doquier a lo largo del siglo XV y no solamente a través de los baldíos como con anterioridad se señaló sino también en perjuicio de las tierras en labrantía proyectándose una reestructuración del espacio agrario.

El cereal sufrirá un desplazamiento tras la reconversión de leiras de heredad eno agro do pam en viñedos ${ }^{28}$ e incluso los prados se orientarán a la producción vitivinícola. $^{29}$

Pero donde la viña se hará fuerte de verdad será en las cortiñas.

La difusión del viñedo por estos espacios $\operatorname{cercados}^{30}$ se expresará con cierta regularidad en la documentación. El poner de viña las diferentes cortiñas será una condición para el cumplimiento del foro tan usual que en todos los monasterios la relación entre estos terrenos y la producción vitivinícola quedará bien patente ${ }^{31} \mathrm{e}$

${ }^{23} \mathrm{CDE}$. Doc 183: ...et he posto que desta dita vina que non labredes mays de tres omees cavadura... e que a tapedes toda arredor como suya estar..., p. 389. También en Docs 180 y 193., C.D.E., pp. 388 y 391.

${ }^{24}$ Doc 190., CDE., p. 391.

${ }^{25}$ CDP. Doc 243: ...vos aforamos o monte do Rego trabeso, que parte con a viña vella de fondo a çima et conmo atravesa per çima da viña de valado a valado..., p. 310.

CDF. Doc 86: ... e dous terreeos que mandou Afonso Anes d-Outer de Vellas ó dito mosteyro, hun jaz envallado, que he de viña, e outro jaz en Outer de Vellas; e mays outros dous leyros de vina que jazen envalado..., p. 107.

${ }^{26}$ CDF. Doc 162: ...vos aforamos a nosa viña que jas ou chaman Caschagoyna ... asy commo está acortinada, per tal pleyto e condiçón que ha ponades de viña de parede a parede..., p. 200.

${ }^{27}$ CDPR. Doc 213: ... outro cortelo de viña en Rogomiro tapado sobre si... de muro e de valado..., pp. 246-247.

${ }^{28}$ Docs 166, 167, 168 y 169., CDE., pp. 384 y 385.

${ }^{29}$ CDP. Doc 88: ...vos aforamos mays, segundo dito he, a leyra que jaz ao prado de viñas con seu monte, per tal pleito et condiçon que a ponades de viña..., p.147.

30 JIMÉNEZ GÓMEZ, Santiago, "Análisis de la terminología agraria en la documentación lucense del siglo XIII" en Actas de las I jornadas de metodología aplicada de las ciencias históricas, vol II, Vigo, Universidad de Santiago ( 1975), pp. 115-133/ 124.

CDF. Doc 163: ...vos aforamos commo dito he toda a nossa cortiña da Freyna, de fondo a çima e de hua parte á outra, de parede a parede, composta e por poer..., p. 201.

${ }^{31}$ CDF. Doc 179: ....Et he posto se aforen a outra cortiña pera poer de viña..., p. 223.

CDF. Doc 212: ... ho noso tarreo, que he do dito noso mosteiro, que jaz, na cortiñs de Masyde... a tal pleyto e condición que ho ponades de viña noba..., p. 271.

Docs 152, 177, 191, 205, 223, 238, 263., CDE., pp. 378, 387, 391, 396, 403, 407 y 415-416.

Docs 118, 120, 142, 174, 302., CDPR., pp. 217, 218-219, 230, 237 у 268.

Doc 14., CDX., p. 177.

CDP. Doc 136: ...Outrosi vos aforamos con esto que dito he a nosa cortyña de Buxeiros, que vos agora teedes posta de viña nova, asi conmo a vos teedes çercada e çerrada de parede..., p. 209. 
inclusive aparecerán como sinónimos ${ }^{32}$. Tanto es así que hasta se instalarán lagares en el seno de estas explotaciones. ${ }^{33}$

\section{Las tierras de cereal}

En esta zona, donde la asociación del viñedo junto al cereal también es importante, tal como acontece en tierras de Melón ${ }^{34}$, las heredades, como término que engloba cualquier extensión de tierra cultivada, y las leiras, referidas a porciones de terreno que junto con otras forman un agro, se constituirán en protagonistas del paisaje destinado a la cerealicultura.

La heredad, recoge un sentido de propiedad plena susceptible de ser transmitida por herencia ${ }^{35}$. Un término que conllevará una gran ambigüedad semántica tal como sentenció Portela Silva ${ }^{36}$ que puede ir desde el conjunto de posesiones pertenecientes a un individuo en una aldea ${ }^{37}$ hasta una parcela ${ }^{38}$. Entre ambos polos opuestos se extenderán un sinfín de situaciones intermedias entre las que se encuentran tanto los terrenos dirigidos a la producción de cereal y de vino ${ }^{39}$

32 CDF. Doc 173: ... conbén a saver que vos aforamos toda a erdade... con a cortiña do Vinnal... a tal pleito e condiçón que lavredes e paredes ben as ditas erdades e voñas commo non falescan con mingoa de lavor e boo paramento..., p. 215.

${ }^{33}$ CDX. Doc 13: ...con seu lagar e os lairatos daa cortiña nova, suo signo de Santa María de Abelenda..., p. 176.

34 RÍOS RODRÍGUEZ, Ma Luz: "El cultivo del cereal en Santa María de Melón (s. XII-XIV)” en II Congreso internacional sobre el cister en Galicia y Portugal: Actas, vol II, Ourense (1999), pp. 749$776 / 753$.

35 JIMÉNEZ GÓMEZ, Santiago, “Análisis de la terminología agraria en la documentación lucense del siglo XIII" en Actas de las I jornadas de metodología aplicada de las ciencias históricas, vol II, Vigo, Universidad de Santiago( 1975), pp. 115-133/ 117.

36 PORTELA SILVA, Ermelindo. "La propiedad, el trabajo y los frutos de la tierra (900-1300)" en Liceo Franciscano( 1978), p. 178.

37 CDP. Doc 252: ... Otrosi mais vos aforamos toda cuanta herdade e casas e arbores e formaes o dito moesteiro ha en Agro Nogueyras, su o sino de santa Maria de Ferreira, con todas suas pertenenças e dereituras que en na dita aldea perteeescan ao dito mosteiro..., p. 319.

${ }^{38}$ En el documento 134 de la colección diplomática de Ferreira el forero en gratitud por el dominio útil recibido emplaza al monasterio. Una heredad de dos tegas de sembradura. Como se verá más adelante esta será aproximadamente la superficie media (entre dos tegas y dos tegas y media de sembradura) que comprenderá una leira. Doc 134., CDF., p. 164.

${ }^{39}$ CDF. Doc 68: ... Outrosí vos damos a nosa herdade que avemos en Goyán... per tal pleyto que a lavredes e a paredes ben e diades dela cada ano ao dito mosteiro terça do pan e meo do vino do que Deus hy der per home do dito mosteiro..., p. 87.

CDE.. Doc 91: ...vos aforamos a nossa herdade... et por tal condiçón que a lavredes et mantenades et paredes bem, et que diades dela cada anno a dita iglesia de Sam Miguel quarta de temporao et terça do serodeo na ayra et quarta de vyno aa byca do lagar... , p. 325. 
como aquellas heredades que comprenden a la vez árboles frutales pastos y dehesas. ${ }^{40}$

Dicho esto, algunos autores identificarán las diferentes heredades con el cultivo exclusivo de cereal ${ }^{41}$ dada la distinción tan manifiesta que se realiza con respecto a las viñas, sotos y cortiñas en el enunciado de los bienes aforados. ${ }^{42}$

La leira es la unidad mínima de explotación en el seno de un agro o heredad que se cultivará de una sola vez y de manera sucesiva ${ }^{43}$, normalmente sin cerco o vallado en la que se sembrarán diversos productos. ${ }^{44}$

La leira, referida a una extensión de cultivo cerealístico, en la mayor parte de las ocasiones, irá ligada al mismo en la estipulación de la renta y no en lo referente a su nomenclatura ${ }^{45}$. De esta forma los escribas monásticos, a diferencia de lo que sucedía con aquellas parcelas destinadas a la producción vitivinícola, prescindirán de cualquier tipo de especificación al respecto. Por tanto, habrá que valorarlas como tierras de labrantío. ${ }^{46}$

Esta afirmación no hará más que corroborarse en la documentación perteneciente a Ferreira de Pantón que es el monasterio que más veces registra términos como leyra o terreos de pan levar o de arameo refiriéndose esta última expresión, según el diccionario de autoridades, a la tierra de labor oportuna para la sembradura siendo esta una voz propia de tierras gallegas. ${ }^{47}$

${ }^{40} \mathrm{CDF}$. Doc 93: ...convén a saber que vos aforamos a herdade que foy de dona Aldara de Temes... e a herdade que deu Roy López ao dito mosteiro... per tal pleito e condiçón que labredes e paredes ben as ditas herdades commo se non percan con mingoa de lavor e de boo paramento, e tenades as froytas e pascos e devissos que perteeçen aas ditas herdades enno dito foro..., p. 115.

${ }^{41}$ LUCAS ÁLVAREZ, Manuel y LUCAS DOMÍNGUEZ: El monasterio de San Clodio do Ribeiro en la Edad Media: estudio y documentos, A Coruña, Edicios do Castro, 1996, pp. 87-88.

42 CDP. Doc 206: ... Conven a saber que vos aforamos todas las casas, viñas, herdades, soutos, arbores, et cortinas que vos mercastes a Gonçalvo da Carreyra..., p. 279.

CDE. Doc 134: ...convén a saber, que vos aforo todas las herdades, e casas, e soutos e árvores e cortinas e propiadades de dizimo a Deus que bos por min tragedes agora a jur e a maao ena dita aldea de Parada e en todos seus términos..., p. 366.

${ }^{43}$ OTERO PEDRAYO, Ramón: Historia de Galiza, vol II, Buenos Aires, Ediciones N ${ }^{\mathrm{os}}$, 1962, p. 176.

${ }^{44}$ LUCAS ÁLVAREZ, Manuel y LUCAS DOMÍNGUEZ, Pedro, El monasterio de San Clodio do Ribeiro en la Edad Media: estudio y documentos, A Coruña, Edicios do Castro, 1996, p. 88.

${ }^{45} \mathrm{CDF}$. Doc 53: ...fazemos carta e damos a foro as nossas leyras destos lugares, as duas leyras jaçem ao chao e jaçen cabo outras leyras do bispo e ontre outras de Sant Adreao, e as outras duas jazen aos campos... per tal preito que vos e a pesoa que pus vos veer que a lavredes e a paredes ben, e se vos deren semente que vos diades meo, se non terça da graa que Deus y der..., p. 68.

${ }^{46}$ RÍOS RODRÍGUEZ, M. ${ }^{a}$ Luz: "El cultivo del cereal en Santa María de Melón (s. XII-XIV)" en II Congreso internacional sobre el cister en Galicia y Portugal: Actas, vol II, Ourense (1999), pp. 749$776 / 753$.

${ }^{47}$ Diccionario de autoridades, Madrid, Gredos, 1976. 
La vinculación de estas explotaciones con el cultivo cerealístico quedará bien definida en los documentos al estar relacionadas las mismas con la estipulación de la renta en centeno o grano ${ }^{48}$ y quedar plenamente diferenciadas de las tierras dirigidas a la producción vinícola. ${ }^{49}$

Junto a heredades y leiras la documentación, aunque de manera exigua, registra la presencia de agras y agros. Seguramente la escasez de referencias respecto a estas tierras de cultivo se deriva a su inclusión en el seno del término mucho más genérico de heredad..$^{50}$

En la actualidad y según el diccionario de la Real Academia Gallega la diferencia entre un agro y una agra resulta bastante evidente. Con el primer término se designa la extensión de tierra para el cultivo, por lo general pequeña, y que pertenece a un solo dueño, mientras que con el segundo, se hace alusión a una gran extensión de tierra de labrantío dividida a su vez en leiras que pertenecen a distintos dueños.

En la época objeto de este estudio esta distinción no será tan diáfana ya que, según Bouhier, estas estructuras se empezarán a consolidar en los últimos decenios del siglo XV y comienzos del XVI. Para este autor, será en este periodo cuando el agro se ordene como una sola pieza de tierra de reducidas dimensiones y cerrada sobre si misma a la vez que la agra o veiga lo hará en forma de espacios mucho más amplios provistos de vallados, paredes o setos que la rodeaban y cercaban mientras que en su interior se encontraban delimitadas por marcos. ${ }^{51}$

Sin embargo, en lo que respecta al área de influencia de los seis monasterios objeto de este estudio, la diferenciación entre ambas estructuras agrarias dista mucho de clarificarse incluso para el periodo señalado por el geógrafo francés. Aunque es cierto que como se ha apuntado las referencias hacia estas explotaciones son escasas todo parece indicar que, en la mayoría de las ocasiones, durante los siglos XIV y XV las palabras agro y agra gozarán de un significado idéntico, es decir, el de una porción de terreno labrado dividido a su vez en varias parcelas, terrenos o leiros.

48 CDF. Doc 199: ...vos aforamos a nosa leyra d-aramio... a tal pleito e condiçón que a labredes e a reparedes ben ho fagades labrar e reparar, e diades e paguedes vos e as ditas vosas vozes a nos $e$ aas que despoys de nos viren enno dito mosteyro de foro e renda de dous en dous annos quatro teegas de çenteo..., p. 250.

CDF. Doc 73: ...e seys tereos d-arameo... per tal preyto e condiçón que os lavredes e os paredes ben e nos dedes cada anno a terça de toda graan que Deus y der..., p. 92.

${ }^{49} \mathrm{CDF}$. Doc 166: ... E por este bem e graça que me vos fazedes enpraço pera sempre ao dito mosteiro dous meus leiros, dízimo a Deus, que eu teño, hun que jaz apar do rego de San Tomé junto con outro do mosteiro que he d-arameo, e outro de viña sobre a fonte de San Tomé..., p. 206.

${ }^{50}$ RÍOS RODRÍGUEZ: "El cultivo del cereal, p. 753.

51 BOHUIER, Abel: Ensaio xeográfico de análise e interpretación dun vello complexo agrario, vol II, s.1., Xunta de Galicia, 2001, pp. 1278-1270/1232-1234. 
Así, en 1300 Martín Mígueles de vende dos leiros en San Martiño de Pantón que iazen en a agra de Cas dos Ferreyros, entre outros de Johán Domínguez de Cas dos Ferreyros $^{52}$, ciento ochenta y tres años después Doña María López, abadesa de Ferreira, aforará outro tarresino que jas ao agroo que parte con outro de Juán de Vilagilde $^{53}$. Queda por tanto bien patente que ambos términos harán referencia a un terreno de labranza dividido a su vez en unidades de explotación menores pertenecientes a diversos dueños. Realidad esta, que toma forma definitiva el 15 de agosto de 1436 cuando el abad Lopo del monasterio de San Esteban de Ribas de Sil afora distintas parcelas (seis leiros y una leira de heredad) situadas en el mismo agro do pan a cuatro matrimonios con el fin que las pongan de viña ${ }^{54}$. El hecho de que el abad exija a uno de estos matrimonios cerrar una leira de heredad dentro del agro ${ }^{55}$ como condición sine qua non para orientarla a la producción vitivinícolahace pensar que estas unidades de explotación destinadas al cereal si bien estaban marcadas y divididas para su clara distinción en el interior del agro se verían desprovistas de cercado ${ }^{56}$ seguramente con el fin, como en la actualidad, de facilitar a los diversos poseedores del dominio útil el libre acceso a las mismas sirviéndose de las parcelas ajenas. ${ }^{57}$

Solamente en una ocasión en 1418 y en San Vicente de Deade, actual municipio de Pantón, se localizarán dos terrenos de agro que responden al rasgo definitorio de pequeñas explotaciones cerealícolas ${ }^{58}$ por lo que seguramente para la mentalidad popular los términos agro o agra se podrían emplear de manera muy diferente.

La constitución de agros o agras no dejará de responder a las estrategias campesinas orientadas a una única finalidad, la de modificar el espacio para que resulte lo más beneficioso posible al individuo. De esta manera, la concentración de diversas parcelas dirigidas a un mismo cultivo garantizará un mejor aprovechamiento de las características edafológicas del suelo así como posibilitará más eficazmente las diferentes labores agrícolas. ${ }^{59}$

52 Doc 29., CDF., pp. 42-43.

${ }^{53}$ Doc 202., CDF., p. 255.

${ }^{54}$ Docs 166, 167, 168 y 169., CDE., pp. 384-385.

${ }^{55}$ Doc 167., CDE., p. 385.

${ }^{56}$ PORTELA SILVA, Ermelindo: La Región del obispado de Tuy en los siglos XII s XV. Una sociedad en la expansión y en la crisis, Santiago de Compostela, El Eco Franciscano, 1976, p. 47.

${ }^{57}$ SÁNCHEZ CARRERA, M ${ }^{\text {a }}$ del Carmen: El Bajo Miño en el siglo XV. El espacio y los hombres, A Coruña, Fundación Pedro Barrie de la Maza, 1997, p. 113.

${ }^{58}$ CDF. Doc 139: ... Outrosí vos aforo mays dous terreos d-agro et de pan levar, hun deles aa Bouça et o outro oo poço do padro, segundo todos jasen sub signo de San Viçenço de Deade...., p. 170.

${ }^{59}$ PALLARES MÉNDEZ, Ma del Carmen y PORTELA SILVA, Ermelindo: El bajo valle del Miño en los siglos XII y XIII. Economía agraria y estructura social, Vigo, Universidad de Santiago, 1971, p. 45. 
Una de las explicaciones que puede llegar a esclarecer la razón de la escasez de menciones tanto de agros como de agras es la que se deriva del proceso de fraccionamiento de estas unidades de producción en otras menores. Tanto es así, que como se ha relatado, los bienes sujetos a foro serán algunas de las divisiones en las que se componen estas extensiones y no la totalidad de las mismas. Con el paso del tiempo, estas porciones de terreno interiores dominarán en lo referente a la documentación al conjunto de las mismas como realidad y como unidades de explotación. ${ }^{60}$

Unidades de explotación cerealística también serán las searas o sernas. Un término que englobará tres significados: el de tierra de labranza en general, tierra señorial y prestación consistente en labrar ${ }^{61}$. De interés en este apartado será el primero de ellos reminiscencia terminológica de la presión a la que se vio sometido el campesinado por los señores desde finales del siglo XI. ${ }^{62}$

Según el diccionario de la Real Academia además de constituir un campo destinado al cereal esta extensión, al igual que sucedía con las agras, estará formada por leiras pertenecientes a diversos propietarios. Este rasgo quedará bien definido en la documentación. ${ }^{63}$

Estas explotaciones, en un número mayor de ocasiones de lo que sucedía con los agros, serán elementos de primer orden a la hora de visualizar el arrinconamiento que sufre el cereal a raíz de la expansión del viñedo sobre todo en la primera mitad del siglo XV. ${ }^{64}$

De hecho la asociación de la seara con la producción vitivinícola será una constante en todas las referencias de la misma a lo largo de la documentación de todo este territorio.

En cuanto a la extensión de las tierras dedicadas al cereal, los datos que nos ofrece la documentación son escasos y fragmentarios, lo que supone una dificultad más a añadir a la complejidad adyacente en la conversión de las medidas de la época al sistema métrico decimal actual. La medida de superficie más utilizada será la teega sementadura, es decir la superficie en la cual se puede sembrar la cantidad de semilla que cabe en una tega de capacidad. Sin embargo, actualmente será el ferrado y no

\footnotetext{
${ }^{60}$ SÁNCHEZ CARRERA: El Bajo Miño en., p. 113.

${ }^{61}$ JIMÉNEZ GÓMEZ: "Análisis de la terminología, p. 123.

${ }^{62}$ GARCÍA DE CORTÁZAR, José Ángel: La sociedad rural en la España medieval, Madrid, Siglo XXI, 1988, p. 101.

${ }^{63}$ CDPR Doc 131: ...damus e outorgamos a foro a vos Fernán Anes... a nossa leyra dEyravedra, que jas a su a nossa seara de Martín Codeyro, así está avalada sobre si...., p. 225.

${ }^{64}$ CDP. Doc 115: ...e outra peça de viña que o dito Iohan Afonso poso en na seara do moesteiro..., p. 187.

CDE.. Doc 130: ...convén a saber, que vos aforamos hun terço da nosa seaara... a tal pleito e condiçón que a ponades de vina nova..., p. 362.
} 
la tega la medida de capacidad utilizada en el área que nos ocupa la cual debido a la mengua de referencias se circunscribirá al actual municipio de Pantón. En todas las parroquias de este ayuntamiento el ferrado de capacidad contendrá 14,72 litros, no muy alejados de los 16 que hace corresponder Bouhier para la tega medieval en Pereiro de Aguiar ${ }^{65}$ que se aproximan a los 15,42 litros estipulados con relación al ferrado en la obra de Fernández Justo para este municipio ${ }^{66}$. Es por ello que una equivalencia tega-ferrado para esta zona no sería una opción descabellada teniendo en cuenta además que en algunos municipios a la tega y el ferrado son la misma cosa. ${ }^{67}$

Pero la dificultad por excelencia a la hora de establecer las dimensiones en metros cuadrados de estos cultivos radica en saber discernir cuanta cantidad de grano era necesaria para sembrar una hectárea de nuestra época hace seis siglos. Para hacer frente a esta disyuntiva he tomado como referencia los 180 litros de grano por hectárea que apunta Sánchez Carrera necesarios para repartir en esos suelos gallegos carentes de un abono adecuado ${ }^{68}$. En la actualidad con las obvias mejoras técnicas de nuestros tiempos la cantidad de simiente utilizada por los labradores para cada hectárea será de 110 litros $^{69}$. Por lo que la cifra utilizada por Sánchez Carrera no debe alejarse demasiado de la realidad inherente al periodo estudiado.

Según expresa, aunque de manera muy sesgada la documentación, las leiras y heredades de cereal comprenderían una superficie de dos a dos y media tegas de sembradura. $^{70}$

Es decir de aproximadamente 817 a $1226 \mathrm{~m}^{2}$. Unas dimensiones que se perpetuarán a lo largo del Antiguo Régimen al ser similares a la extensión de la parcela media en diversas localidades lucenses estudiadas por Villares Paz para el siglo XVIII. ${ }^{71}$

Una cuestión a destacar es que solamente se especificará el tamaño de estas explotaciones cuando el receptor de las mismas sea el monasterio por medio de

\footnotetext{
${ }^{65}$ BOHUIER: Ensaio xeográfico, vol II, p. 1289.

${ }^{66}$ FERNÁNDEZ JUSTO: La metrología tradicional, vol I, p. 461.

${ }^{67}$ Ibidem, pp. 89-90 y 473.

${ }^{68}$ SÁNCHEZ CARRERA: El Bajo Miño en, p. 121.

${ }^{69}$ Según las informaciones extraídas de labradores residentes en el municipio de Pantón.

${ }^{70} \mathrm{CDF}$. Doc 134: ... Et poreste ben e graçia que eu recibo do dito mosteiro enpraço e dou ó dito mosteiro a mia erdade que jas a Villa da Careyra.... que seerá dúas teegas sementadura...., p. 164.

CDF. Doc 204: ... Et posto este ben e merçede que vos recibo et do dito voso mosteiro vos implazo de enprazo a miña leira do Chao que he dicimo a Deus, que son dúas tegas semente...., p. 259.

CDP. Doc : ...vendemos a vos don Afonso Gonçales, prior do moesterio de San Viçenço de Pombeiro... huna leyra de herdade a que chaman de Ribeyra con seu terreo, que son duas teegas e medea de pan sementadura. Outrosy vos vendemos mays con esto que sobredito he outra leyra a que chaman do Salgueyro e con outra leyra de Coto de Rey, que seeran anbas çinquo teegas de semente...., p. 197.

${ }^{71}$ En concreto en tierras del Salnés, Burón y Friol. VILLARES PAZ, Ramón, La propiedad de la tierra en Galicia 1500-1936, Madrid, Siglo XXI, 1982, p. 25.
} 
emplazamiento o venta. De ahí se infiere el inconveniente de establecer una metrología más extensa relativa a las tierras de cultivo ya que la mayor parte de la documentación para este periodo, como es sabido, se refiere a contratos de aforamiento donde es el forero el destinatario de los bienes.

\section{PRÁCTICAS AGRÍCOLAS}

\section{El cuidado del castaño y de la vid}

Los cuidados que requerían tanto el cultivo del castaño como el de la vid lo hacían de este una verdadera práctica de jardinería.

Una de las condiciones más recurrentes en la documentación del monasterio de San Vicente de Pombeiro será la obligación impuesta al forero por los rectores monásticos de plantar nuevos castaños ${ }^{72}$. Junto a este requisito y con el fin de que estas especies arbóreas alcanzasen los niveles exigidos de productividad, serían necesarias ciertas labores manifestadas sólo superficialmente a lo largo de la documentación. Entre ellas destacarán como actividades básicas para el buen cuidado de los sotos la poda y el injerto. ${ }^{73}$

Ambas prácticas como es lógico suponer se orientaban a mejorar el aprovechamiento de los castañares. Así la poda y el injerto cubrirían un doble objetivo, por un lado incrementar la cualidad del fruto y por otro la utilización del ramazón sobrante como parte del combustible calefactor del hogar familiar ${ }^{74}$ junto con la madera de roble. Además, tras estas labores la copa fructífera de los castaños lograba un mayor despliegue en detrimento de la altura de los mismos que sería menor que la de las especies silvestres. De esta forma las castañas eran de una mejor calidad e incluso el forero podría establecer una gradación en el cultivo según injerte variedades prematuras o tardías. ${ }^{75}$

72 CDP. Doc 93: ... et que ponades hy dez castineiros que prenda de garfeo et de rays...., p. 151.

73 CDP. Doc 176: ... et roçedes et podedes ben o dito souto commo se non se pergan por mingoa de lavor et de bon paramento...., pp. 250-251.

CDE. Doc 255: ... que ponades en cada hun anno despoys de mina vida quatro castineyros $e$ enxertedes aqueles que foren para enxertar... ., p. 413.

CDP. Doc 54: ...per tal pleyto que o chantedes de souto, e çepedes e enxertedes os ditos soutos cada hun u vir [a que] lo for mester... ., p. 108.

74 PICALLO FUENTES, Héitor: "Castañas e castiñeiros como recursos económicos na Galiza do medievo" en Raígame: Revista de arte, cultura e tradicións populares, Ourense, Diputación de Ourense, 18 (2003), pp. 57-74/ 58.

75 RÍOS RODRÍGUEZ, Ma Luz: "Soutos bravos y soutos mansos: el castaño en Galicia (siglos XII-XIV)"en El medio natural en la España medieval: Actas del I congreso sobre ecohistoria e historia medieval, Cáceres, Universidad de Extremadura (2001), pp. 475-488/ 482. 
En cuanto a la recolección de las castañas, nada deja entrever la documentación. Sin embargo, es más que posible que esta actividad no haya diferido mucho a lo largo de siete siglos. Así, durante las últimas semanas de septiembre y finales del siguiente mes se recogerían las especies precoces que una vez ya maduras se hubiesen precipitado al suelo. En cambio para las variedades tardías se emplearía el vareo de las ramas para conseguir un mayor número de frutos ${ }^{76}$. Una vez realizada la recolección, las castañas constituyen parte de la renta monástica. En la mayoría de las ocasiones en las que los castaños estaban sujetos a renta proporcional de su cosecha esta la recogerían los agentes del monasterio justo a pie del bien aforado ${ }^{77}$ en su variedad de castañas verdes ${ }^{78}$. No obstante, los señores se inclinaban más por las castañas secas produciéndose su reparto bien en las cisternas, bien en canizos y sequeiros ${ }^{79}$. Sería lógico suponer que prevaleciese en la documentación una correspondencia entre castañas verdes-renta proporcional y castañas secas-renta fija. Esta última circunstancia, en relación con las castañas secas, se dará siempre en los censos pertenecientes a los monasterios de San Pedro de Rocas y de San Esteban de Ribas de Sil. Pero tal reciprocidad se difuminará al cruzar el Sil. De esta manera en el monasterio de Pombeiro las castañas secas tanto pueden ser objeto de renta fija o proporcional indistintamente ${ }^{80}$, pero eso sí, linpas de moyña, o lo que es lo mismo sin cáscara.

El cultivo de la vid, a su vez, requeriría una serie de cuidados que se extendían a lo largo de todo el año, atenciones en extremo necesarias ante la posibilidad de que la viña no produjese lo previamente estimado por los rectores monásticos ${ }^{81}$.

Las labores ya empezaban en invierno mediante una labranza con el fin de romper los terrones y airear las raíces resguardándolas de las aguas estancadas ${ }^{82}$,

\footnotetext{
76 PICALLO FUENTES, Héitor: "Castañas e castiñeiros como recursos económicos na Galiza do medievo" en Raígame: Revista de arte, cultura e tradicións populares, Ourense, Diputación de Ourense, 18(2003), pp. 57-74/ 66-67.

77 CDPR. Doc 125: ...e darnos edes quinta das castanas que Deus y der ao toro do castineiro..., p. 221.

${ }^{78} \mathrm{CDF}$. Doc 36: ...e dardes as meas das castanas que en el ouver des que foren coleytas verdes..., p. 51.

${ }^{79}$ RÍOS RODRÍGUEZ, Ma Luz: "Soutos bravos y soutos mansos: el castaño en Galicia (siglos XII-XIV)"en El medio natural en la España medieval: Actas del I congreso sobre ecohistoria e historia medieval, Cáceres, Universidad de Extremadura (2001), pp. 475-488/ 484.

${ }^{80}$ CDP. Doc 43: ... et os quatro moios de castanas secas e linpas de moyno a deçeda do caniço..., p. 95.

CDP. Doc 78: ...et terça de castañas a deçeda do canyço, secas et linpas de moyña..., p. 138.

${ }^{81}$ CDP.Doc 39: ... et se esta leyra minguar de vinna que vennan a estaren..., p. 91.

82 CANTERA MONTENEGRO, Enrique: "Instrumentos y técnicas de cultivo en la plena Edad Media europea (siglos X-XIII)" en Cuadernos de Historia Medieval, Madrid, 8 (1987), p. 53.
} 
después se estercolaba la tierra. Si la viña era ex novo obviamente, una vez estercolado el terreno se procedía a plantar los bacelos o viña nueva ${ }^{83}$ necesitados a largo de dos o tres años de una serie de atenciones semejantes a las que recibían las vides en producción. ${ }^{84}$

Entre estos cuidados la documentación claramente hace referencia a la poda que se hacía y hace entre los meses de enero y febrero, aunque también es común que tras la vendimia en otoño se procediese a desmochar las varas sobrantes. La poda constituía una de las prestaciones en trabajo que el forero ha de realizar para el monasterio de San Pedro de Rocas ${ }^{85}$. De esta forma se sabe que la poda como servicio duraba sólo un día ${ }^{86}$. Tras la poda se ligaban las vides a estacas de madera, apostandolas $^{87}$, con el fin de dotarlas de la forma definitiva que les permita una mejor orientación al sol. ${ }^{88}$

En el transcurso del año se efectuaban de dos a tres cavas. En invierno y en primavera con el fin de efectuar un abonado y entrechantar de viña nueva las cepas viejas con el fin de renovarlas ${ }^{89}$ y en verano para despojar de las viñas las malas hierbas surgidas tras las lluvias primaverales. ${ }^{90}$

Ya en septiembre tenía lugar la más sustancial labor de la viticultura, la vendimia y la posterior vinificación de la uva.

Estrictamente en relación con la vendimia la documentación solamente se hace eco del férreo control que ejercen sobre esta actividad los monjes y sus agentes.

Así, la documentación no se vuelve cicatera a la hora de mostrar las obligaciones que ha de cumplir el campesinado en esta última fase del trabajo en la viña.

${ }^{83}$ CDE. Doc 142: ... a tal pleito e condiçón que ponades a dita leyra de Forcadas de vina nova en estes dous annos primeyros segentes..., p. 374.

CDP. Doc 81: ... a tal pleito et condiçon que poñades de baçelos a dita leira en estes quatro annos primeiros seguentes..., p. 141.

${ }^{84}$ RODRÍGUEZ GALDO, Ma Xosé: Señores y campesinos en Galicia. Siglos XIV-XVI, Santiago de Compostela, Pico Sacro, 1976, p. 94.

85 Docs 261, 269, 271, 285 y 286., CDPR., pp. 258, 260, 261 у 264.

86 Docs 278, 283, 284, 294., CDPR., pp. 262, 263-264 y 266.

${ }^{87}$ CDP. Doc 178: ...e elas postas de viña que as labredes e reparedes e apostedes todas ben en tal maneira que non desfallezcan con myngua de lavor e de boo paramento..., p. 253.

88 ÁLVAREZ ÁLVAREZ, Eleutino: "El cultivo de la vid en la Tierra de Lemos en el siglo XV" en Jubilatio. Homenaje de la facultad de Geografía e Historia a los profesores D. Manuel Lucas Álvarez y D. Ángel Rodríguez, González, vol I, Santiago de Compostela, Universidad de Santiago (1987), pp. 149-159/ 157.

${ }^{89}$ CDP. Doc 109: ... a tal pleito e condiçon que a entrechantedes (una leira de viña) de vina nova en estes tres annos primeyros seguentes, se lle nesçesario for..., p. 181.

${ }^{90}$ CDP. Doc 229: ...per tal pleito et condiçon que britades as matelas que en ela estan..., p. 298. 
Ineludiblemente éste ha de pedir permiso al monasterio para poder iniciar la vendimia ${ }^{91}$ bajo la atenta mirada de un enviado suyo ${ }^{92}$. En algunas ocasiones los señores, incluso, reclamaban una pequeña renta cuando el forero pidiese para vendimiar. ${ }^{93}$

Una vez realizada la vendimia las uvas son trasladadas al lagar para iniciar un proceso de vinificación del que los documentos poco se preocupan. Si muestran mucho más interés, una vez convertida la uva en vino, en reflejar con claridad el acopio de la renta por parte del enviado del monasterio. Este agente señorial, que tenía que ser alimentado a costa del forero, tenía la misión de "medir" y "partir" lo correspondiente a los monjes según las condiciones del foro ${ }^{94}$. Este reparto se hacía frecuentemente en el mismo lagar o bodega donde el forero había elaborado el vino ${ }^{95}$ ya que estos anexos, aunque de propiedad monástica, se circunscribían, al igual que la tierra, al dominio útil del campesino como bienes aforados que $e^{2}{ }^{96}$. Esta circunstancia queda bien reflejada a la hora de incluir en el contrato disposiciones por las que se exigía al forero dejar colocar las cubas de los señores en el interior de dichas instalaciones. ${ }^{97}$

El interés señorial no sólo se ceñía al vino también lo haría en relación con la composta, humus del viñedo que se empleaba como fertilizante ${ }^{98}$ y que a diferencia de la uva quedaba en la viña para ser recogida bajo la vigilancia del agente del cenobio ${ }^{99}$. Otro tipo de modalidad relativa a la exacción de renta documentada, aunque mucho menos frecuente, era la que en vez de ejecutarse en el lagar se rea-

${ }^{91}$ CDF. Doc 134: ...e non vindemedes sen mandado do dito mosteiro..., p. 164.

CD.R. Doc 213: ... mais nos dares nosa vista quando quiserdes vindimiar..., p. 247.

CDP. Doc 258: ...et viredes demandar a vindimia et liçençia para coler os ditos nobos..., p. 324.

92 CDF. Doc 162: ...e que chamedes ó noso mayordomo quando collerdes o vino..., p. 200.

CDE. Doc 104: ... et quando quiserdes vindymyar que chamedes ao ovençal..., p. 336.

${ }^{93}$ CDE. Doc 107: ...et que diades hun moravedí de pan quando quiserdes vendimyar..., p. 339.

CDF. Doc 215: ... E da cortyna e terreos pagaredes en quada hun anno durante boso foro e personas darnos ees por bysta de byndymya hua galyna e más vyndymya, e más alende que por dya da nosa festa que nos beñás honrar con do que teberdes..., p. 275.

${ }^{94}$ CDP. Doc 270: ... a oytaba de todo o viño, medido o dito viño por lo moordomo do mosteiro ao qual daredes de comer..., p. 337.

CDE.. Doc 122: ... e chamedes para midir o dito vino e para o partir ao noso mordomo ao qual proveeredes de comer e de beber quando se mydir o dito vyno..., p. 353.

$95 \mathrm{CDE}$. Doc 91: ...et quarta de vyno aa bica do lagar pelo granieyro da dita iglesia ou por seu home..., p. 325.

CDP. Doc 172: ...que dian quinta do vino que Deus en nas viñas dier, todas a a bica do lagar, per ollo de noso mayordomo..., p. 247.

${ }^{96}$ CDF. Doc 148: ....Et outrosí vos aforamos mays a casa do lagar que he do dito conbento..., p. 181.

${ }^{97} \mathrm{CDE}$. Doc 143: ... vos aforamos hua nossa casa de adega... a tal pleito e condiçón... que leixedes poer en ela hua cuba cada hun anno ao convento..., p. 375.

${ }^{98}$ LÓPEZ CARREIRA: "O traballo da viña..., p. 161.

${ }^{99}$ CDPR. Doc 108: ...et nos dedes delo cada ano o noso moesteiro quinta de todo novo e froito que Deus y der, o vino no lagar e a composta na vina, por noso moordomo, o qual os quaes vos sobre ditos devedes a proveer de comer e de beber comunalmente mentre colerdes o novo do dito lugar..., p. 210. 
lizaba en las viñas mismas donde se "partían” las uvas en cestos bajo la atenta vigilancia del mayordomo monástico. ${ }^{100}$

Una vez seleccionada la cantidad de vino que el forero había de satisfacer para colmar las exigencias de la renta le atañía a éste, en unas ocasiones, el transporte de la misma a la bodega del monasterio ${ }^{101}$, mientras que en otras sería el representante señorial quien se encargue de la faena ${ }^{102}$ seguramente ante la tendencia del forero de "rebajar" el vino con agua ${ }^{103}$ circunstancia que requería estrechar la supervisión por parte del cenobio. ${ }^{104}$

\section{El trabajo en tierras de cereal. El sistema de cultivos.}

Para empezar a tratar las prácticas agrícolas relativas al cereal hay que partir de dos supuestos muy diferenciados entre sí.

El primero hace referencia a la tesis unánimemente aceptada por la historiografía francesa que afirma que tras la segunda mitad del siglo XIII se hizo necesaria la intensificación de los cultivos con el fin de subsanar el detenimiento que sufrió el proceso de conquista de nuevas tierras y así, hacer frente a la continua demanda de víveres. En este nuevo modelo de rotación, las leguminosas cobrarán gran relevancia en detrimento del barbecho. ${ }^{105}$

El segundo se extrae de la proposición de Jesús García Fernández que sostiene que tal intensificación, en Galicia, no tendría cabida antes del siglo XVIII y aún en esta centuria el cultivo intensivo no adquirirá carácter de generalidad en todo el país ni en las áreas donde se estableció estaría bien conseguido. ${ }^{106}$

100 CDF. Doc 194: ...et daredes a quinta do viño que Deus der nas ditas viñas, as uvas enna viña partidas per los çestos per ollo de noso mayordomo segundo custume..., p. 243.

CDF. Doc 215: ... e nos dyás en quada hun anno da dita cortina e terreos a quarta das uvas que Deus der en elas, partidas por los cestos por ollo de noso moordomo ou de nosos certo requado..., p. 275.

101 CDF. Doc 32: ...e medeo de viño aa bica di lagar en salvo, et tragérdelo ao moesteiro por vossa custa..., p. 46.

CDP. Doc 148: ...e trageredes todo o noso quinon do viño e dos outros novos per vosa custa a a nosa adega..., p. 224.

102 CDPR. Doc 316: ... ao mordomo quando for saquar lo vino e algún se for con el..., p. 271.

103 CDF. Doc 200: ...e nos pagaredes de foro por todo elo en vosa vida hun moyo de vino puro e sen augoa e sen maao sabor por día de San Martino de novembro en cada un anno..., p. 252.

CDF. Doc 176: ...e que nos diades cada hun anno vos e as ditas persoas ao dito convento dous canados de vino por dia de San Martino de noviembro, que seja vino syn agua e syn malicia..., p. 219.

104 CDE. Doc 242: ...dos canados de vino sen agua o espeto da cuba por ollo de noso mordomo..., p. 409 .

105 DUBY, Georges: Economía rural y vida campesina en el Occidente medieval, Barcelona, Altaya, 1999, pp. 134-136.

106 GARCÍA FERNÁNDEZ, Jesús: Organización del espacio y economía rural en la España Atlántica, Madrid, Siglo XXI, 1975, p. 111. 
Por su parte, tanto Portela Silva como Pallares Méndez en sus respectivos estudios acerca de la región del obispado de Tuy y del monasterio de Sobrado ${ }^{107}$ defienden, siguiendo el modelo de Duby, la teórica existencia de dos modelos de actuación intensiva que desplazarían el año de barbecho a los periodos estrictamente necesarios para evitar el agotamiento de los suelos. Lo que dilucidaría el empleo de un barbecho irregular. El primero de estos modelos, se basaría en la siguiente alternancia:

- siembra del cereal de primavera en marzo - cosecha del cereal de primavera en agosto - supresión del barbecho y preparación de la tierra para la segunda siembra - siembra del cereal de invierno en octubre - cosecha del cereal de invierno en agosto - periodo de reposo de seis a siete meses - siembra del cereal de primavera en marzo y vuelta a empezar.

En el segundo entrarán en escena las leguminosas y será el que sigue:

- siembra del cereal de primavera en marzo - cosecha del cereal de primavera en agosto - reposo hasta marzo - siembra de leguminosas en marzo - cosecha de leguminosas en agosto - preparación de la tierra durante dos meses siembra del cereal de invierno en octubre - cosecha del cereal de invierno en agosto - seis o siete meses de reposo - siembra del cereal de primavera en marzo y vuelta a empezar.

En el primero de los dos tipos es obvio pensar que el riesgo de disminución de la cosecha por agotamiento hará preceptivo la utilización de un barbecho discontinuo en el tiempo y variable en su duración. El segundo esquema será mucho más racional ya que la distribución de los periodos de reposo de la tierra y el cultivo de leguminosas favorecerá la oxigenación y fertilidad del suelo.

Pese a ello Portela Silva argumenta que la difusión del primer modelo, el que conlleva la eliminación drástica del año de barbecho regular, se generalizaría sobre todo por aquellas comarcas, como la que ocupa este estudio, donde la reducción de las tierras productoras de pan en favor de aquellas destinadas al cultivo del vino lo hizo indispensable. ${ }^{108}$

No obstante, la consulta de los diferentes estudios regionales, cuestiona de manera clara el predominio de los sistemas de cultivo intensivos dentro del panorama agrario medieval gallego.

107 PALLARES MÉNDEZ, M ${ }^{\mathrm{a}}$ del Carmen: El monasterio de Sobrado: un ejemplo de protagonismo monástico en la Galicia medieval, A Coruña, Diputación Provincial de A Coruña, 1975, pp. 166-168.

PORTELA SILVA, Ermelindo: La Región del obispado de Tuy en los siglos XII s XV. Una sociedad en la expansión y en la crisis, Santiago de Compostela, El Eco Franciscano, 1976, pp. 125-128.

108 PORTELA SILVA: "La propiedad, el trabajo y, p. 199. 
Redundando esta línea de interpretación Ríos Rodríguez, por su parte, tomará una posición más ecléctica en la relación con el predominio de un sistema de cultivo u otro en tierras de Melón. Para esta autora tanto el barbecho y la rotación de cultivos como inclusive la intensificación propuesta como predominante por Portela serán complementarios y nunca excluyentes entre sí. ${ }^{109}$

En lo que respecta al señorío de Meira, Mariño Veiras hace compaginar en este territorio el sistema bienal junto con otros modelos más extensivos (como el cultivo al tercio) con el fin de mantener un equilibrio entre la ganadería y la agricultura. ${ }^{110}$

También en el dominio del priorato de Vilar de Donas, que se extendía por el corazón de Galicia ocupando parte de las actuales cuatro provincias gallegas, se observa como incluso durante la segunda mitad del siglo XV el sistema de año y vez no dará paso a la rotación trienal. ${ }^{111}$

Por último el estudio de Rodríguez González centrado en las no muy lejanas tierras del Bierzo hará hincapié en la rotación bienal como forma predominante por lo menos en lo que respecta al área de influencia del monasterio de San Andrés de Espinareda. ${ }^{112}$

Como se podrá apreciar seguidamente en la Ribera Sacra medieval tampoco parece claro que la intensificación de cultivos y la supresión del barbecho se instituyese como un indiscutible arquetipo de cultivo prevaleciente sobre el resto.

La gran dificultad para establecer el sistema de rotación de los cultivos se encuentra en el hecho de que en la totalidad de los foros, las rentas que comprenden los escasos cereales de primavera y legumbres documentados están relacionadas con la explotación de casales, lugares y heredades diversas. Estas unidades de explotación constituirán un conjunto de tierras que no sólo no tienen por que explotarse al mismo tiempo sino que cabe la posibilidad de que inclusive estén separadas entre sí. Es esta circunstancia la que hace que la investigación sobre las prácticas agrícolas del campesinado medieval gallego se torne cuesta arriba. La producción de un casal o de diferentes heredades no determinará su procedencia de un mismo terrazgo sometido a un único sistema de explotación en el que los cultivos

${ }^{109}$ RÍOS RODRÍGUEZ: "El cultivo del cereal en, p. 754.

110 MARIÑO VEIRAS, Dolores: Señorío de Santa María de Meira (De 1150 a 1525). Espacio rural, régimen de propiedad y régimen de explotación en la Galicia medieval, La Coruña, Ediciones Nos, 1983, p. 288.

111 NOVO CAZÓN, José-Luis: El priorato santiaguista de Vilar de Donas en la Edad Media (1194-1500), A Coruña, Fundación Pedro Barrie de la Maza, 1986, p. 146.

112 RODRÍGUEZ GONZÁLEZ, Ma Carmen: Economía y poder en el Bierzo del siglo XV. San Andrés de Espinareda, Santiago de Compostela, Universidad de Santiago, 1992, p. 107. 
se suceden $\sin$ interrupción ${ }^{113}$. Esta cuestión se esclarecería si el objeto mayoritario del foro estuviese integrado por las leiras que como únicas porciones de terreno cultivado reflejarían con claridad el ciclo de cultivos anual. Pero este no será el caso.

De hecho, las exiguas apariciones en los textos de los cereales de primavera junto a las leguminosas, en comparación con el centeno o el trigo, no hacen sino abrir la posibilidad de la permanencia en esta época de un modelo de producción de cereal de invierno cada dos temporadas. Esta observación tomará forma si se tiene en cuenta que el cultivo de la cebada aparecerá incluso en el seno de las huer$\operatorname{tas}^{114}$, es decir, en un tipo de terreno de un valor y significado distinto al relativo al terrazgo donde supuestamente se ejercería el ciclo de cultivos. De esta forma, que la renta de casales, lugares y herdades conste de centeno, trigo y mijo o cebada no significará necesariamente que estos cereales se siembren en una misma unidad de labor y por tanto, que el rasgo definitorio de estas explotaciones sea el policultivo.

La presencia del barbecho anual estará documentada en el área de nuestro estudio por medio del foro de una leira de aramio por la renta de una talega de centeno cada dos años ${ }^{115}$. Así pues, esta parcela estaría en barbecho al menos un año hasta que volviese a ser productiva. No obstante, hay que advertir que el documento abre la posibilidad de que dicho contrato pueda llegar a reconvertirse en subforo ${ }^{116}$ privilegiando a sus receptores, habitantes del arrabal de Monforte y probablemente burgueses, con una renta mínima cada dos años en vez de la pertinente anual.

También se aprecia una renta fija de grano cada dos años en el documento 78 de la colección diplomática de Ferreira117. Aunque aquí, en la heredad aforada, el objeto principal de la renta es el vino siendo la porción de tierra dedicada al cereal mínima y por ello, cabe la posibilidad que la renta exigida por la misma no pase de ser un mero reconocimiento hacia el monasterio en vez de un claro exponente de la productividad de este terreno.

Curiosamente, las tierras sujetas a este tipo de censos en sendos casos se circunscribirán en el entorno de hábitats concentrados, como corresponde en el primer caso en al arrabal del mismo Monforte y en el segundo a la aldea de Goyán.

113 PORTELA SILVA, Ermelindo: La Región del, pp. 124-125.

114 CDF. Doc 50: ...salvo dúas ortas que som a Souto de Pantón con súa çeveyra..., p. 65.

115 CDF. Doc 199: ... vos aforamos a nosa leyra d'aramio... de foro e renda de dous en dous annos quatro teegas de çenteo..., p. 250.

116 Ibidem: .... a tal pleito e condiçón que a labredes e reparedes ben ho fagades labrar o reparar..., p. 250.

${ }^{117} \mathrm{CDF}$. Doc 78: ...et dardes de dous en dous anos hua teega de pam per teega derreyta de Monforte..., p. 98. 
El cultivo bienal también se documentará en la modalidad de sistema de rotación de año y vez. Es decir, la división de los terrenos en dos hojas, una de las cuales era sembrada de cereal de invierno mientras que la otra, de menor extensión, se dedicaba al barbecho, alternando la disposición al año siguiente. Así, en 1477 se aforará una heredad en los dominios del monasterio de San Esteban de Ribas de Sil a condición de que el forero pague ena folla que se labrar coarta de todas graas ${ }^{118}$. El hecho que solamente una hoja se dedicase a la labranza y no dos pone de manifiesto aún en este periodo tardío la perseverancia de la rotación bienal frente al sistema de dos cultivos cada tres años, perseverancia que también se dejará entrever en la documentación de San Vicente de Pombeiro. ${ }^{119}$

La perduración en los siglos finales de la Edad Media del barbecho anual y de la rotación bienal en el área que ocupa este estudio no puede extrañar si se tiene en cuenta la recopilación realizada por Lucas Labrada del informe del corregidor de Monterrey fechado en 1803 en el que se expresa que incluso la tierra más fértil de esta villa, aún en esta época, está reducida en la mayor parte a dar un fruto de trigo o centeno cada dos años. ${ }^{120}$

Redundando en esta proposición y ya en el marco de la Ribera Sacra, concretamente en el ámbito de las Tierras de Chantada, Villares Paz argumentará para la segunda mitad del siglo XVIII que en términos rigurosos, el barbecho sistemático - una cosecha de cereal de invierno con descanso aproximado de quince meses-se sigue en el 93 por 100 de las tierras labradías, con escasas diferencias zonales, siendo, pues, el "cultivo con intermisión” o bianual la técnica que utiliza el labrador del interior en su trabajo de las tierras no fertilizadas por la bondad del río Miño. ${ }^{121}$

Así, este protagonismo del cultivo de año y vez aún en periodos tan tardíos contrastará directamente con los condicionantes básicos, ensalzados por parte de la historiografía de la Europa atlántica ya para la alta Edad Media, que condicionan el progreso agrario y económico oriundo solamente del septentrión del continente y completamente ajeno del extrarradio mediterráneo ${ }^{122}$ tan arcaico y rudimentario. Estos condicionantes, que estarán estrechamente ligados a la propagación de la idea

\footnotetext{
118 Doc 230., CDE., p. 405.

119 CDP. Doc 266: ... e pagaredes quanto labrardes a a folla da herdade do dito mosteiro disemo enteyro da dita granja de santa Mariña..., p. 332.

${ }^{120}$ LUCAS LABRADA, José: Descripción económica del Reino de Galicia, Vigo, Galaxia, 1971, p. 123.

${ }^{121}$ VILLARES PAZ, Ramón: La propiedad de la tierra en Galicia 1500-1936, Madrid, Siglo XXI, 1982, p. 27.

122 WHITE, Lynn: Tecnología medieval y cambio social, Barcelona, Paidós, 1990, pp. 93-95.
} 
que asevera que la agricultura extensiva puede ser objeto de un paulatino desarrollo de la productividad de los campos mediante labores más intensas ${ }^{123}$, se manifestarán en forma tanto de adelantos tecnológicos ${ }^{124}$ como de una gradual transición de la rotación bienal a la trienal e introducción de leguminosas. ${ }^{125}$

Sin embargo, como opina Fossier el poner resistencia a aceptar un progreso determinado no indica siempre el atraso, la rutina o el egoísmo; ciertas condiciones sociales pueden justificar un rechazo e incluso a menudo la oposición a una novedad señala más lucidez o previsión que adoptarla sin discusión. ${ }^{126}$

Opinión esta que encontrará eco en Barceló quien argumenta que la agricultura extensiva medieval no será más que un proceso de mengua continuada de la feracidad de la tierra en el que solo la recuperación de la misma mediante el barbecho o la práctica de rozas esporádicas por fuego del monte (es decir las prácticas en teoría más "primitivas") comportarán la única solución concebible para el campesinado. Así, las distintas innovaciones técnicas orientadas a la intensificación del cultivo tendrán su origen no en la eficacia del campesinado sino en la irracionalidad de la renta feudal más preocupada en hacer accesible lo producido que en aumentar la producción. ${ }^{127}$

Es más, tras la crisis del XIV la gran disponibilidad de tierras permitiría al campesinado labrar las diferentes parcelas durante un periodo determinado extraer de ellas un par de cosechas y dejarlas descansar dos, tres o incluso cuatro años. El bajo índice demográfico daría la oportunidad a los supervivientes a labrar un número mayor de tierras por lo que la rotación trienal y la intensificación de la agricultura serían consideradas una mala estrategia ante la alternativa mucho más ventajosa de poder trabajar parcelas beneficiadas por largos periodos de reposo. ${ }^{128}$

De esta manera, el barbecho no habrá que juzgarlo ni mucho menos como un sistema técnico propio de poblaciones primitivas y atrasadas sino como el pilar esencial sobre el que se cimientan de todas las posibles aquella estrategia de acorde el entorno físico y humano más eficaz para conseguir una cantidad mayor de producción de cereal panificable precisamente gracias al año de descanso ${ }^{129}$.

${ }^{123}$ BLOCH, Marc: La sociedad feudal, Madrid, Akal, 2002, p. 92.

${ }^{124}$ DUBYuby, Georges: Hombres y estructuras de la Edad Media, Madrid, Siglo XXI, 1993, p. 161.

125 WHITE, Lynn: Tecnología medieval y cambio social, Barcelona, Paidós, 1990, pp. 85-93.

${ }^{126}$ FOSSIER, Robert: La infancia de Europa. Aspectos económicos y sociales, Barcelona, vol II, Labor, 1984, p. 488.

127 BARCELÓ, Miquel et alii: Arqueología medieval en las afueras del "medievalismo", Barcelona, Crítica, 1988, pp. 219-221.

${ }^{128}$ LALIENA CORBERA, Carlos, "Tecnología agraria medieval en medios semiáridos" en Técnicas agrícolas, industriais e constructivas na Idade Media. Curso de verán, Celanova, 8-12 de xullo de 1996, Santiago de Compostela, Tórculo (1996), p. 99. 
De hecho, una de las características de cualquier sociedad es la de no cambiar algo por una novedad si este algo aún cumple satisfactoriamente la función que dicha sociedad le ha encomendado.

En cuanto a las labores agrícolas empleadas por el campesinado para sacar un mayor rendimiento a sus tierras hay que decir que encontrarán un escaso eco en la documentación monástica.

Obviamente en primer lugar se hacía necesario una correcta fertilización de los suelos. En un periodo en el que el abono de origen animal era escaso debido a la extensión de las tierras ganadas al cereal y a la limitada estabulación del ganado, ya que el porcino y bovino pastaba en el monte, por lo que su estiércol se desperdiciaba ${ }^{130}$ se hacía necesario recurrir a las variedades vegetales. Entre estas la de mayor relevancia por su uso a través de los tiempos por el campesinado gallego destacará el tojal. ${ }^{131}$

Esta especie solamente es referida en la documentación del monasterio de Ferreira relativa al siglo XV. No deja de ser extraña la circunstancia si tenemos en cuenta que en este periodo muchas zonas de bosque sometidas a desbroces continuados y a un periódico pastoreo se ven impedidas a recuperar sus formaciones arbóreas originales dejando paso al dominio del matorral ${ }^{132}$. Además será frecuente la repoblación de estas zonas baldías de tojales destinados tanto a la elaboración de abono como a la alimentación del ganado. La parvedad del tojal en la documentación no hace más que ratificar la monopolización del interés de las fuentes sobre aquello susceptible de generar rentas.

Las diferentes colecciones diplomáticas solamente se referirán dentro de las labores agrícolas a aquellas destinadas a la recolección del cereal, es decir, la siega y la malla.

Como sucedía con la vendimia el forero tendría que pedir permiso para efectuar tales actividades ${ }^{133}$ que se debían de realizar bajo la supervisión de un representante monástico ${ }^{134}$. Era este quien en la era ${ }^{135}$, una vez el grano quedase separado de

\footnotetext{
${ }^{129}$ RÍOS RODRÍGUEZ: "El cultivo del cereal en..., p. 755.

${ }^{130}$ CANTERA MONTENEGRO, Enrique: "Instrumentos y técnicas de cultivo en la plena Edad Media europea (siglos X-XIII)" en Cuadernos de Historia Medieval, Madrid, 8 (1987), p. 55.

${ }^{131}$ RÍOS RODRÍGUEZ, $M^{\mathrm{a}}$ Luz: "Transformación agraria. Los terrenos de monte y la economía campesina (s. XII-XIV)" en Torres Luna M $\mathrm{M}^{\mathrm{a}}$ del Pilar et alii, Espacios rurais e sociedades campesiñas, Santiago de Compostela, Universidad de Santiago de Compostela, 1997, pp. 145-172/ 150-151.

132 GUTIÁN RIVERA, Luis y PÉREZ ALBERTI, Augusto: Historia ecológica de Galicia, Santiago de Compostela, Universidad de Santiago, 2002, p.118.

133 CDPR. Doc 110: .... Outrosí non segedes nen maledes sen nos..., p. 211.

CDP. Doc 250: ... et quando quiserdes malar viredes a demandar liçença ao dito prior ou ao dito mordomo..., p. 218.

134 CDPR. Doc 119: ...todo por noso mordomo, o qual vos e as ditas vozes proveerdes de comer et de bever comunalmente, e devedes a chamar para segar e caregar et malar..., p. 218.

135 CDP. Doc 70: ...e daredes quarta de pan en a eyra, todo per noso ome..., p. 129.
} 
la cáscara y de la paja, condición indispensable para que este se constituyese en renta $^{136}$, procedía a repartir entre el forero la cosecha ${ }^{137}$. En cuanto al traslado de la renta, independientemente de su estipulación como fija o proporcional , en las más de las ocasiones sería una tarea a realizar por un agente señorial ${ }^{138}$ aunque tampoco sería del todo anómalo ver sometido a esta carga al forero. ${ }^{139}$

\section{* SIGLAS Y ABREVIATURAS}

CDE - DURO PEÑA, Emilio: El monasterio de San Esteban de Ribas de Sil, Orense, Instituto de Estudios Orensanos "Padre Feijoo", 1977.

CDPR - DURO PEÑA, Emilio: El monasterio de San Pedro de Rocas y su colección documental, Orense, Instituto de Estudios Orensanos "Padre Feijoo", 1972.

CDF - FERNÁNDEZ DE VIANA Y VIEITES, José Ignacio: Colección diplomática del monasterio de Santa María de Ferreira de Pantón, Lugo, Diputación provincial de Lugo, 1994.

CDC - FERNÁNDEZ SUÁREZ, Elisa: "El monasterio de Santa Cristina de Ribas de Sil”en Boletín Auriense, 4, (1974), pp. 7-66.

CDP - LUCAS ÁLVAREZ, Manuel y LUCAS DOMÍNGUEZ, Pedro: El priorato benedictino de San Vicenzo de Pombeiro y su colección diplomática en la Edad Media, A Coruña, Edicios do Castro, 1996.

CDX - PEREIRA FERREIRO, Sara: "El monasterio de Santa María de Xunqueira de Espadañedo y su colección diplomática" en Boletín Auriense, 9, (1979), pp. 151-230. 333.

136 CDE. Doc 100: ...e que nos diades del... hua fanega de çenteo linpo de poo et de palla..., p.

137 C.D.E. Doc 159: ...chamaredes ao seo moordomo para mallar e para partir e medir dos ditos novos..., pp. 382-383.

138 C.D.E. Doc 124: ... et que diades del cada hun anno en vosa vida a quinta parte do pan que Deus y der, et despoys de vosa morte que dian quarta de pan, et todo esto daredes ao oveençal da vistiaria..., p. 355.

C.D.F. Doc 81: ...convén a saber tres quarteiros de çenteo e hun quarteiro de serodeo, per teega dereita de Monforte ou da Prova... e diades de comer ó mordomo que for por lla renda..., p. 102.

C.D.E. Doc 88: ...hun moyo de çenteo e tres quarteyros de seródeo lynpoo... et dardes de comer ao home que for recadar essa renda..., p. 320.

139 C.D.E. Doc C.D.E Doc 74: ...e levardes o nosso quinón y ao nosso celleyro por vos..., p. 308.

C.D.F. Doc 36: ...e adugeredes o pan per vossa custa ao dito mosteyro..., p. 51.

C.D.F. Doc 65: ...e avedes de adozer o noso quinón da graa per vosa custa ó noso çelleyro do mosteiro..., p. 83. 